\title{
Rejecting Behaviour and Separation Initiatives as Aspects of the Mother-infant Independence Dynamics in Rhesus Monkeys
}

\author{
Tartabini Angelo \\ Department of Neuroscience, University of Parma, Italy
}

Copyright (C) 2015 by authors, all rights reserved. Authors agree that this article remains permanently open access under the terms of the Creative Commons Attribution License 4.0 International License

\begin{abstract}
This paper suggests that attachment, rejecting behaviour and independence initiatives among rhesus monkeys (Macaca mulatta) constitute the most critical elements of mother-infant interactions. Results of previous studies of different family groups indicate that both the mothers' rejecting behaviour and the infants' independence initiatives (or social activities) are not homogeneus but instead differ from each other, depending on subjects' individual differences and on the diverse relationships and experiences among members of the family groups. These findings underline the relevance of a methodology that takes into account individual, dyadic and social variables among subjects. This complex of variables represents a potentially useful methodology for studies on the attachment-independence process in mother-infant interactions in rhesus monkeys and in many other species of monkeys.
\end{abstract}

Keywords Independence, Rejection, Rhesus Monkeys, Separation

\section{Introduction}

Attachment behaviour is a dyadic relationship that is an essential characteristic of both human and non-human Primates from the first day of life until death. Moreover, attachment is a necessary condition for the development of the independence of the infant. As a classification of behaviour, attachment is the maintenance of the infant's proximity to the mother.

Attachment is vital for the survival of offspring. The infant needs secure attachment, that is an attachment in which it is confident that the mother figure is consistently available and helpful, especially in dangerous situations. Moreover, secure attachment involves the mother's understanding of the dyad's social context, kinship and her readiness to intervene when necessary to protect the infant. With this secure attachment, an infant will be confident in exploring the environment and social interactions away from its mother $[1,2,3]$.

In the healthy growth of offspring, monkeys, humans and many other mammals must develop a strong, self-confident attitude that the infant acquires by staying in close contact with the mother. A secure attachment dissipates fear and permits exploration. An insecure attachment gives rise to fearful behaviour. Fearful behaviour occurs in strange situations and when the mother is at a certain distance from the infant. A secure attachment that the infant establishes with the mother allows him/her to leave her temporarily in order to socialize with the other members of the group and to practise some fundamental physical activities, such as social play behaviours with same-age infants. Secure attachment followed by independence also allows the infant to discover his/her capability of social investment and his/her role in the group [10].

Virtually at birth, the rhesus infant has all the necessary physiological capacities which allow him/her to grip the mother's fur. Through evolution, humans have lost this ability. However, this loss has been substituted by greater maternal attention and care toward the child $[4,5,6]$. Also, among different species of monkeys body contact between mother and offspring develops differently and results in differences in offspring care. For instance, an infant rhesus monkey grips onto the mother in a variety of different positions even if the most common one is the ventro-ventral position. The gripping positions change very frequently, especially when the infant reaches three or four months [10]. The basic behavioural categories in mother-infant interaction are body contact and rejecting behaviour.

Fundamentally behavioural components considered are: ventro-ventral contact with or without nipple contact; rejecting behaviour, that is control of nipple contact by the mother;

- and the infant's social interaction, including play behaviour, with other group members after being rejected $[7,8,9,10,11]$. 


\section{Rejecting Behaviour Literature}

The mother-infant rejecting behaviour in non-human primates has been observed and studied by other authors even if the bibliography is till now numerically limited [12, $13,14,15,16,17,18,19]$. The topic has always been seen as a function of a certain number of social variables, such as the mother's needs in her role in the group, the offspring's age, and the mother-infant conflict.

The above cited literature concerning rejecting behaviour reveals that:

1) The offspring is rejected by the mother especially when the infant has achieved enough physical and motor activity and the mother is annoyed by this activity around her or on her body. Moreover, another cause of the rejecting behaviour is hypothesized as the possibility that the mother introduces her son or daughter into the social activity of the group and farther away from her when the offspring reaches a certain age.

2) The mother rejects her infant without violence (otherwise it is abuse and its cause should be found elsewhere) and she encourages him/her to stay separated from her (Off mother) in order that the infant may initiate some social activity and begin to acquire some fundamental competence for his/her future. This latter way of keeping the infant away from mother, which we call mother-infant rejection, is almost always a subtle movement by the mother. But there are some exceptions to this. On occasion the mother rejects the infant without considering the consequences that may occur as a result of the separation. Some of these consequences may be very serious for the offspring and instead of a rejection stimulating self-confident behaviours, it may inhibit the infant.

3) When the infant is rejected with moderation and if he is in the presence of other infants, above all same-age infants, he/she looks immediately for social contact. In this case, same-age infants in the same group will have an influence on rejected infants. The rejected infant takes and receives many initiatives of play behaviours.

\section{Body Contact Dynamics}

The body contact dynamics between mother and infant in monkeys develop according to the morphological growth of the infant. For instance, in the first few weeks of life for the rhesus monkey when the mother leaves her infant, the infant is not passive toward this initiative. The infant tries to rejoin his/her mother. This includes following the mother and trying to recontact her body. In the first phases of the infant monkey's development, we can see that after the mother takes the initiative of leaving her offspring while he/she was in the ventro-ventral position and with the nipple in the mouth, the infant follows her, quietly or moaning, until he/she achieves his/her purpose of rejoining her. The mother who is engaged in such a behaviour interrupts the suckling and also breaks body contact with the offspring. Afterwards the body contact is a physical contact and it is a way of feeding the offspring, as well as something more important than this. That is, it is a way of protecting and comforting the infant, as well as a way of decreasing the tension and conflict between the dyad. In monkeys it is amid such a mixture of behaviours involving body contact that conflict arises between mother and infant [20,21, 22].

\section{Rejecting Behaviour}

Primate mother-infant conflict is characterized by social behaviour that focuses on the mother's rejecting behaviour and the infant's response to it. Rejecting behaviour is the most critical phase of the attachment independence process. This behaviour arises when the infant has already established and consolidated an attachment with his/her mother and when the mother starts seeking independence from the offspring. The mother seeks independence because she wants to be free to pursue her other maternal duties, such as protecting or taking care of other infants in her family. Another important pursuit of the mother is contact with other adult members of the group for mating or other social purposes.

Mother-infant conflict often becomes acute during the mating season, as was found in Gelada baboons Theropithecus gelada when the duties of infant care hinder the mother's social relations with adult males for reproductive purpose [21]. Conflict arises when the mother begins to limit the infant's access to her nipple. In rhesus monkeys some data $[5,6]$ indicate that the reduction of access to the mother's nipple increases dramatically at the age of about three or four months, a little earlier than the four to five months reported by Gomendio [20].

Gomendio also considered the influence of physiological factors. For instance, during the mother's oestrus the infant increases its attempts to access the nipple, while the mother increases her rejecting behaviour, creating a conflict in the dyad. Between three/four months and about eight months low rejection is correlated with a decrease in suckling due to the fact that the infant himself/herself is seeking more independence at this age $[20,22]$.

\section{Mother Passive Prevent (MPP)}

Rejecting behaviour is more properly called Mother Passive Prevent (MPP) [4]. The mother prevents her infant from contacting her breast, interposing between her and her infant one arm to prevent his/her approaching and reaching for the nipple. This passive prevent sometimes takes place before the infant reaches ventro-ventral contact. Another similar behaviour pattern occurs when the mother turns her body away from the infant who is trying to approach the mother. In conclusion, the rejecting behaviour is the mother's 
manifestation of indisposition toward her offspring's attempts at contact.

It is the manifestation of an open conflict between mother and offspring in which the two subjects have opposite wishes. The mother tends to follow her need for independent movements, and the infant tends toward maintaining contact with the mother for comfort, protection and feeding (milk). It must be remembered that the infant at this age (before weaning) still needs and depends on the mother.

Another dynamic category that has to be considered in the analysis is the social interactions in which the infant (by then separated from mother, actively or passively) is involved with other members of the group: e.g., adult male, brothers, sisters (sisters with their own offspring or without ofspring), cousins and aunts. For social interaction, the initiative of the contact has to be taken into consideration. The contact is of two kinds: Initiatives of the mother-infant interaction and initiatives of social contact with all other members.

Regarding the initiative of the mother-infant interaction, the observations should initiate when the mother and the infant are physically separated and at a distance of a minimum of $60 \mathrm{~cm}$ (Off mother). On the other hand, in social contact with same-age infants or with the other members of the group, a distinction has to be made between the contact initiative flowing into play behaviour or as a simple body contact. The initiative of contact among infants with all other members of the group has to be considered too [23, 24].

It is necessary to investigate the bodily contact between infant and mother, especially the difficulties that emerge during the development of the offspring in which he/she desires contact with the mother for as long as possible and the mother, instead, attempts to reduce the length and the frequency of the contact. In rhesus monkeys, for example, these problems arise after the infant is about three months of age.

To approach the study of the mother-infant interaction dynamics in the first few weeks of infant development and in the early phase of weaning, some specific questions need to be considered:

a) The first question concerns the nature of the mother-infant interaction in rhesus monkeys and especially before the period in which the infants are partially or totally independent from the mothers. What is the role of the two partners regarding the initiatives that tend toward the separation of the dyads and, in the opposite direction, tend toward the rejoining of the dyads?

b) Since the mother-infant relationship is not a rigid phenomenon but is modified and developed over a period of time and changes with the morphological growth of the offspring, is the mother mainly responsible for the control of the relationship with her son or daughter? From the first day of life until weaning, does the offspring take an absolutely passive role in the relationship with his/her mother?

c) In monkeys, but also in many other animals, individual characteristics are important factors. All subjects have their own

personality that result from their relationship with their mothers and all other members of the group. Also, the personality is the way in which each infant develops its characteristics, acting with all the others. Since such individual differences exist in the management of the mother-infant relationship, how can the mother or the infant intervention affect the management of the relationship itself?

\section{Final Discussion}

One argument arising from the results already obtained [see $5,6,11$ ] is that the infants' social activities are rather different from each other, especially in the cases in which the social contact is preceded by maternal rejection. This means that we have to dismiss the idea that rejecting behaviour has a generic effect on the social behaviour of all infants when they are far from their mothers. In fact, the case of the daughter of a dominant mother is emblematic. The maternal rejection in this subject drives her to increase social activity with the other members of the group, especially with her elderly sister who does not have any sons and daughters. This is less clear in an another subject infant who has two sisters in her group.

It can be confirmed the idea that with maternal rejection the infant's initiatives with others are less rather than more playful, although the overall rates of social contact initiatives may not be less frequent $[5,6]$. Moreover the maternal rejection does not depend on the fact that the infants were creating a nuisance by a high rate of playing. The mother inhibits the infants' social play especially in the cases in which the infants perform high [and not low] percentages of play. If the rejected infant tries to contact the other members of the group, instead of his/her mother, then the consequences of the rejection depend on how disposed partners are toward social contact $[25,26]$. The situation becomes quite difficult when the rejected infant does not find, or does not like to contact, the other members of the group and he/she insists on contact with the mother.

Finally, Rejecting behaviour and Nipple contact have an effect on the frequency of body contact between mother and infant. In fact, Nipple contact assuredly opposes the breaking of the body contact while the rejecting behaviour decreases the infants possibilities of reaching the nipple. In the dominant mother, the rejecting behaviour blocks the nipple contact especially within five seconds of the beginning of contact. This is less evident in subordinate mothers [11].

The conclusion is that infants at three/four months have the ability to understand when and how to contact their mothers to reach the nipple and when the mothers are ready or not ready to accept contact. It can be confirmed that Mother Passive Prevent (MPP) toward infants affects infant social interactions and relationships within the group. This influence apparently is not homogeneously distributed among our subjects. In some case, Mother Passive Prevent greatly reduced the percentage of the infant play behaviour. In contrast, in another subject the effect could be significantly the opposite. The conclusions could be that 
Mother Passive Prevent has different effects on different subjects within different families, relationships and experiences, even if the general tendency is that the Mother Passive Prevent reduces the initiatives of social contact with other members of the same group.

Is there any relationship between Mother Passive Prevent and playful initiative taken by rejected infants according to their potential social companions? From the results it seems that Playful initiatives are drastically reduced when the rejected infants directed their attention to older companions, compared with cases in which they are directed towards same-age infants.

In conclusion individual differences among our subjects are evident. The Mother Passive Prevent generally reduced play differently among the subjects, but particularly reduced play among infants with a previously higher rate of play. Also, the consequences of rejecting behaviour depend on the kind of companions available in the groups and family composition.

The mother does not reject randomly and does not reject the male more than the female offspring, but that the rejecting behaviour can be correlated to the composition of the group (e.g., presence/absence of individuals such as brothers and sisters). In fact the rejected infant sometimes seeks comfort from companions but this depends on the availability of suitable companions. Also, the rejected infants who persist in trying to contact their mothers because there are no alternative companions (or because they do not want companions), may suffer for it and lose confidence. Finally, it can be supported the fact that to establish confidence, particular attention has to be paid to the social and environmental context in which the rejection occurs.

\section{Acknowledgements}

I am grateful to the Italian CNR (Centro Nazionale delle Ricerche) and the British Council for supporting for several years my research and collection data at the rhesus monkey colony of the MRC Unit on the Development and Integration of Behaviour at Cambridge University in Madingley (U.K). I am also gratefull to Dr. Michael Simpson and Prof. Robert Hinde of the MRC Unit provided in the past inestimable assistance and cooperation.

\section{REFERENCES}

[1] Hinde, R.A. 1974. Biological bases of human social behaviour. New York: McGraw-Hill Inc.

[2] Hinde, R.A. 1979. Towards understanding relationships. London: Academic Press.

[3] Bowlby, J. 1988. A secure base: parent-child attachment and healthy human development. London: Routledge.

[4] Tartabini, A. \& Simpson, M.J.A., 1986. The use of confidence intervals in arguments from individual cases: maternal rejection and infant social behaviour in small groups of rhesus monkeys. In: G.J. Else and P.C. Lee, Ontogeny, Cognition and Social Behaviour of Primates, Cambridge: Cambridge University Press, pp.: 239- 249.

[5] Tartabini, A. \& Simpson, M.J.A., 1987. Maternal rejection and infant play in rhesus monkey infants. Int. J. of Anthropol., 2(1): 21-27.

[6] Tartabini, A. \& Simpson, M.J.A., 1987a. Reactions of infant rhesus monkeys (Macaca mulatta ZIMMERMAN) to the mother's rejecting behaviour: A methodological approach. Monitore Zoologico Italiano, N.S., 21: 201-202.

[7] Berman, C.M., 1980. Mother-infant relationships among freeranging rhesus monkeys on Cayo Santiago: A comparison with captive pairs. Animal Behaviour, 28: 860-873.

[8] Berman, C.M., 1990. Intergenerational transmission of maternal rejection rates among free-ranging rhesus monkeys. Animal Behaviour, 39: 329-337.

[9] Chapais, B. \& Berman, C.M. 2004. Kinship and Behavior in Primates. New York: Oxford University Press.

[10] Tartabini, A., 1991. Social play behaviour in young rhesus monkeys Macaca mulatta at three different ages: from the 3rd to the 6th month of life. Behav. Process., 24: 185-192.

[11] Simpson, M.J.A. \& Tartabini, A., 1992. Control of nipple contact by mothers and infants in rhesus macaques. Folia Primatol., 59: 26-32.

[12] Hinde, R.A. \& Simpson, M.J.A., 1975. Qualities of motherinfant relationships in monkeys. In: ASP Elsevier (Editor), Parent-Infant Interaction. Amsterdam: Ciba Foundation Symposium 33: 39-67.

[13] Simpson, M.J.A., 1985. Effects of early experience on the behaviour of yearling rhesus monkeys Macaca mulatta in the presence of a strange object: classification and correlation approaches. Primates, 26: 57-72.

[14] Simpson, M.J.A. \& Simpson, A.E., 1986. The emergence and maintenance of interdyad differences in the mother-infant relationships of rhesus macaques: a correlational study. Int. J. of Primatol., 7: 377-397.

[15] Simpson, M.J.A. \& Howe, S., 1986. Group and matriline differences in the behaviour of rhesus monkey infants. Animal Behaviour, 34: 444-459.

[16] Simpson, M.J.A., Simpson, A.E. \& Howe, S., 1986. Changes in the rhesus mother-infant relationship through the first four months of life. Animal Behaviour, 34: 1528-1539.

[17] Simpson, M.J.A. \& Howe, S., 1980. The interpretation of individual differences in rhesus monkey infants. Behaviour, 72(3-4): 127-155.

[18] Simpson, M.J.A. \& Datta, S.B., 1990. Predicting infant enterprise from early relationships in rhesus macaques. Behaviour, 116(1-2): 42-63.

[19] Hopkins, W.D. \& De Lathouwers, M. 2006. Left nipple preferences on infant Pan paniscus and Pan troglodytes. International Journal of Primatology, 27(6): 1653-1662.

[20] Gomendio, M., 1991. Parent/offspring conflict and maternal investment in rhesus macaques. Animal Behaviour, 42: 993-1005. 
[21] Dunbar, R.I.M. \& Dunbar, R., 1988. Maternal time budgets of gelada baboons. Animal Behaviour, 36: 970-980.

[22] Hinde, R.A. and White, L.E., 1974. Dynamics of a relationship: Rhesus mother-infant ventro-ventral contact. $J$. of Comp. and Physiol. Psychol., 86: 8-23.

[23] Simpson, M.J.A., 1979. Problems of recording behavioural data by keyboard. In: M.E. Lamb, S.J. Suomi and G.R. Stephenson (Editors), Social Interaction Analysis: Methodological Issues, Wisconsin: University of Wisconsin Press, pp.: 137-156.

[24] Hinde, R.A. \& Herrmann, J., 1977. Frequencies, durations, derived measures and their correlations in studying dyadic and triadic relationships. In: H.R. Schaffer (Editor), Studies in Mother-Infant Interaction, London: Academic Press, pp.: 19-46.

[25] Simpson, M.J.A. 1991. On declaring commitment to a partner. In: P.P.G. Bateson (Editor), The Development and Integration of Behaviour. Essay in honour of Robert Hinde, Cambridge: Cambridge University Press, pp.: 271-293.

[26] Simpson, A.E. \& Simpson, M.J.A., 1985. Short-term consequences of different breeding histories for captive rhesus macaque mothers and young. Behav. Ecol. Sociobiol., 18: 83-89. 